\title{
SUR UN GASTEROSTOMIDE IMMATURE CHEZ SINIPERCA
}

\section{Par TSENG SHEN}

Mon collègue, l'ichthyologiste Hsien-Wen Wu, m'a aimablement remis des viscères de Siniperca scherzeri (Basil.), dans lesquels il avait remarqué la présence de parasites. Le Siniperca parasité provenait de Chung-King (Province de Sétschouen, Chine). J'ai soumis ces viscères parasités à l'examen de R.-Ph. Dollfus, qui y a découvert trois espèces de trématodes digénétiques et a bien voulu me guider dans l'étude de l'une d'entre elles, qui appartient à la famille des Gasterostomid $x=$ Bucephalidæ. Je me fais un devoir de remercier R.-Ph. Dollfus pour tous les précieux conseils qu'il m'a donnés.

Rappelons brièvement que la systématique des Gasterostomata, actuellement en usage, dérive de celle établie par Odhner (1905) qui, le premier, a nettement défini le groupe et a réparti les espèces alors connues dans les deux genres Gasterostoma von Siebold sensu str. et Prosorhynchus Odhner, d'après la structure de leur organe de fixation, occupant l'extrémité antérieure du corps. Chez Gasterostoma von Siebold (Odhner, 1905 sensu), l'extrémité antérieure est en ventouse ; chez Prosorhynchus Odhner, elle est en rostellum.

W. Nicoll (1914), ayant repris l'étude des Gasterostomata, proposa de diviser Gasterostoma von Siebold (Odhner, 1905 sensu) en trois genres :

Bucephala von Baer, 1827, pour les espèces à organe antérieur pourvu de tentacules musculaires rétractiles (fimbriæ).

Bucephalopsis Diesing, 1855, pour les espèces à organe antérieur consistant en une simple ventouse globuleuse.

Rhipidocotyle Diesing 1858, pour les espèces à organe antérieur comprenant une ventouse à faible musculature, surmontée d'un lobe contractile en éventail. Pour ces trois genres, Nicoll a proposé la sous-famille des Bucephalinæ Nicoll, 1914. Pour le genre Prosorhynchus Odhner, Nicoll a proposé la sous-famille des Prosorhynchinæ Nicoll, 1914.

Nicoll, comme auparavant Poche (1907), a rejeté le genre Gasterostoma von Siebold, 1848 au profit de Bucephala von Baer, 1826, conformément à la loi de priorité, qui s'applique bien que Buce-

Annales de Parasitologie, T. VIII, $\mathrm{N}^{\circ} 5 .-1^{\text {er }}$ octobre 1930 , p. 554-561. 
phala ait été créé pour une forme larvaire. Plus récemment, Y. Ozaki (1924 et 1927) a révisé les Gasterostomata, conservant les deux sous-familles établies par Nicoll mais ajoutant trois genres nouveaux : Gotonius Ozaki, 1924 dans les Prosorhynchinæ; Nannoenterum Ozaki, 1924 et Dolichoenterum Ozaki, 1924, dans les Bucephalinæ, ce qui portait le nombre total des genres à sept. Un hutième a été, depuis lors, proposé par R.-Ph. Dollfus : Prosorhynchoïdes $\mathrm{R} .-\mathrm{Ph}$. Dollfus, 1929, pour une espèce à long intestin formant une arcade entre la face ventrale et la face dorsale, puis se prolongeant postérieurement, le long de la paroi dorsale, jusqu'à l'union des dèx derniers tiers de la longueur du corps, ou un peu au-delà.

Le gastérostomide de Siniperca a son extrémité antérieure pourvue d'un rostre épineux. je le rapporte au genre Prosorhynchus Odhner et je dédie l'espèce au Prof. Clément Vaney, de la Faculté des Sciences de Lyon, en souvenir de son bon accueil.

\section{Prosorhynchus vaneyi $\mathrm{n}$. sp.}

Corps allongé, plus pointu postérieurement qu'antérieurement, long de 580 à $840 \mu$, large de 160 à $220 \mu$, à cuticule entièrement couverte de spinules. Appareil antérieur de fixation long d'environ $90 \mu$, large d'environ $57 \mu$, très rétractile ; en extension, il montre une triple couronne d'épines cunéiformes, nettement plus grosses que les spinules (longueur $3 \mu$ sur largeur $1 \mu 5$ environ), qui garnissent toute la surface du corps.

Les épines de la triple couronne sont en quinconces, celles de la rangée moyenne alternant avec celles des deux autres. On remarque que les épines de la rangée moyenne sont plus grandes et plus grosses $(6 \times 12 \mu)$ que celles des deux autres. Toutes les épines ont leur pointe dirigée postérieurement. La musculature du rostre est bien visible, on distingue : $1^{\circ}$ des faisceaux larges d'environ $3 \mu$ s'insérant d'une part sur le disque, d'autre part sur la paroi latérale de l'appareil terminal et servant à la rétraction. $2^{\circ}$ au-dessous de la couronne, des faisceaux (larges d'environ 4 à $5 \mu$ ) de fibres annulaires limitant une cavité en cupule, comblée par des cellules glandulaires environnées de tissu mésenchymateux. La bouche est située sur la face ventrale vers l'union du tiers moyen et du tiers postérieur du corps. Le pharynx a un diamètre de $60 \mu$ et sa musculature radiaire est assez fortement développée. L'intestin, en forme de poche, est long de $105 \mu$, large de $81 \mu$; il est séparé du pharynx par l'œsophage, qui mesure environ $30 \mu$ de long. L'ensemble du tube digestif a son axe dirigé antérodorsalement à partir de la face 


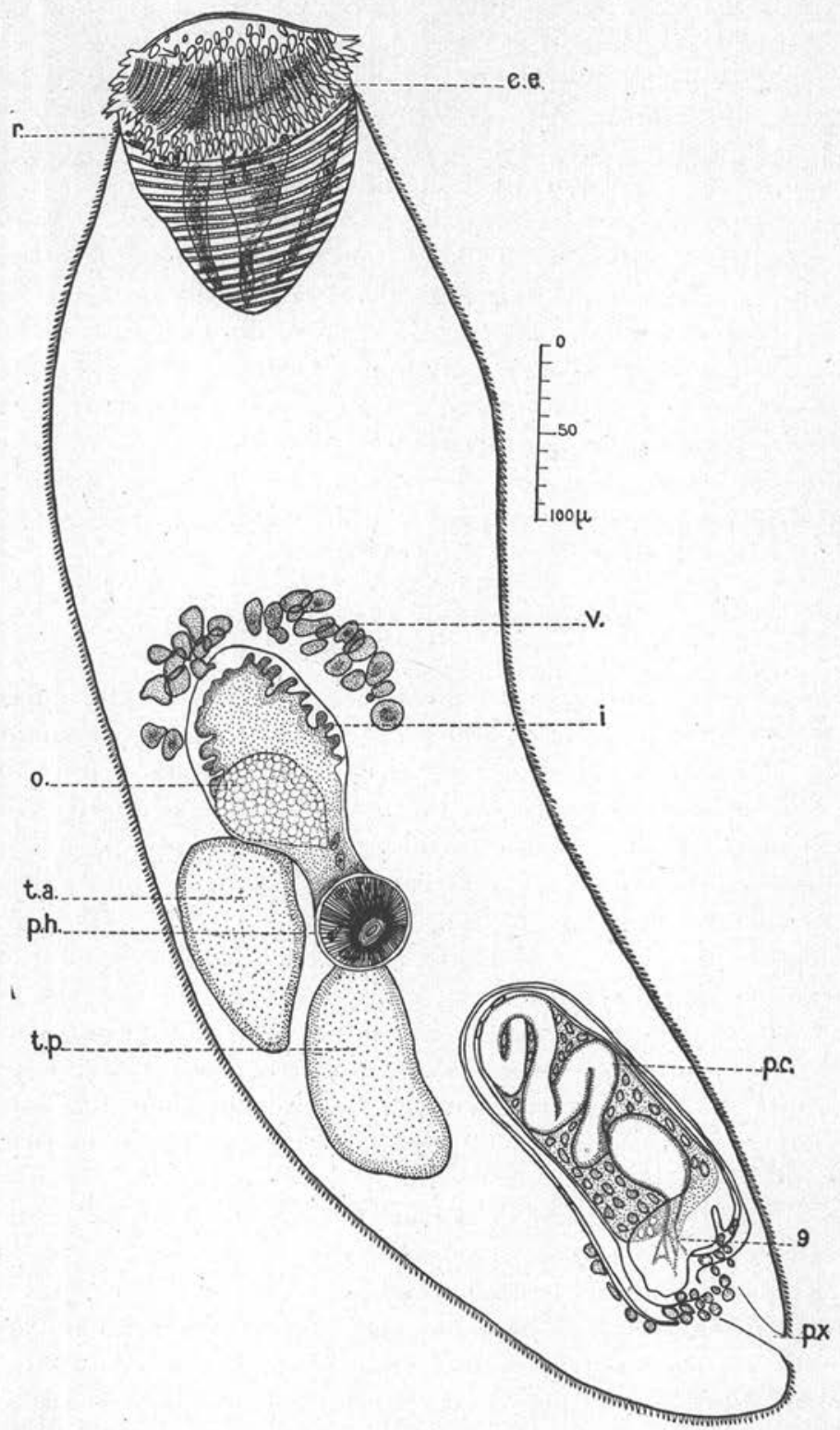

Fig. 1. - Prosorhynchus vanegi n. sp., vu par la face ventrale : $c e$, couronne d'épines ; $r$, rostre ; $v$, vitellogènes ; $i$, intestin ; $o$, ovaire ; $t a$, testicule antérieur ; $p h$, pharynx ; $t p$, testicule postérieur ; $p c$, poche du cirre ; $g$, Geschlechtszapfen ; $p x$, pore excréteur. 
ventrale, à peu près dans le plan sagittal du corps. Le fond de l'intestin atteint antérieurement à peu près la limite entre les deux premiers tiers de la longueur totale du corps, où se trouve la concavité de l'arc formé par les follicules vitellogènes. Dans la paroi de l'œsophage et de l'intestin on voit des cellules glandulaires et épithé-

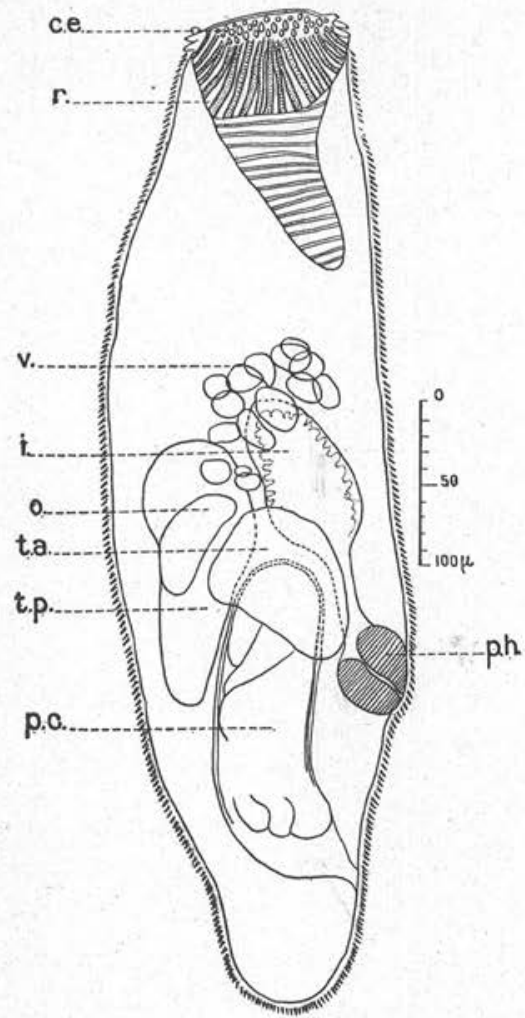

Fig. 2. - Vue de profil montrant les rapports des organes. Le rostre n'est pas complètement étendu (mêmes lettres).

liales, la surface de l'épithélium du sac intestinal, présente des villosités.

Les vitellogènes sont composés d'un petit nombre (au maximum une trentaine) de follicules séparés, plus ou moins ovales ou arrondis, mesurant de 9 à $10 \mu$ sur 15 à $18 \mu$; ils sont disposés suivant un arc à concavité postérieure, embrassant le fond de l'intestin. L'ovaire est situé à un niveau intermédiaire au pharynx, au fond de l'intes- 
Caractéristiques des espèces

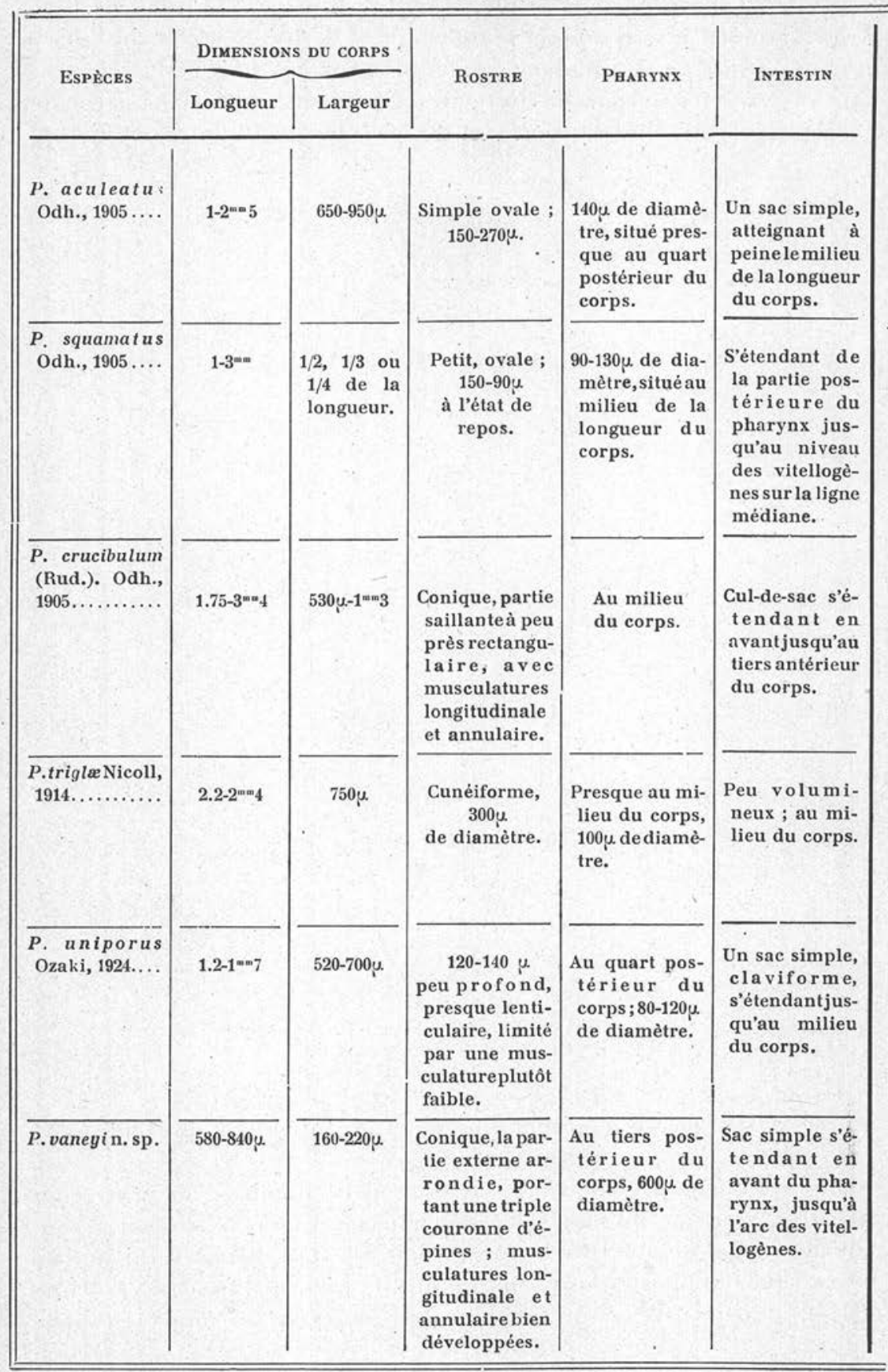




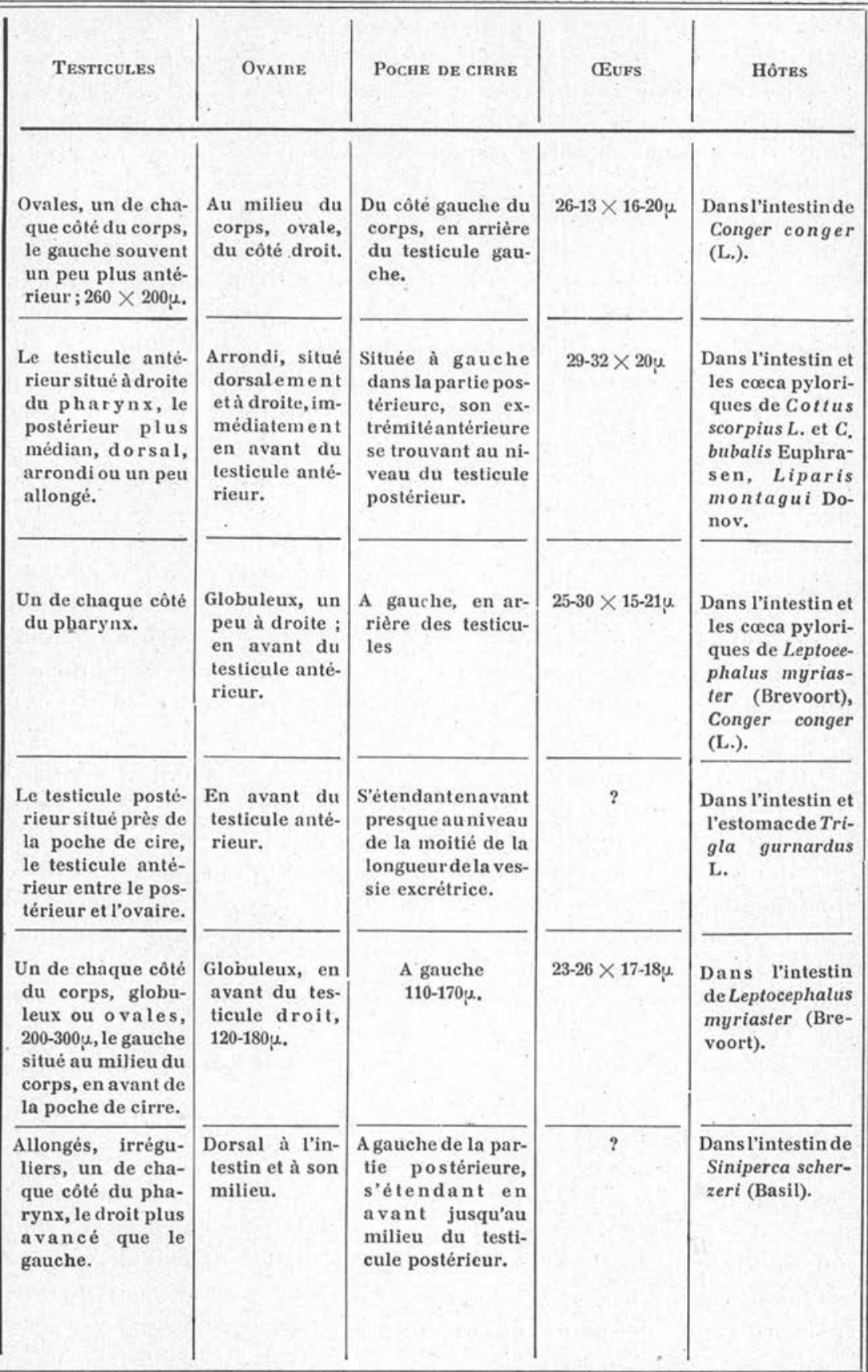


tin et masqué par lui lorsque l'on regarde l'animal par la face ventrale ; sa forme est ovale, il mesure environ $63 \mu$ sur $48 \mu$. Les testicules ont une forme irrégulière et sont plus longs que larges, leur position n'est pas absolument fixe : l'antérieur, à droite du pharynx, atteint antérieurement l'ovaire; le postérieur, plus à gauche, atleint antérieurement le pharynx et dépasse postérieurement le fond de la poche du cirre.

La poche du cirre mesure environ $186 \mu$ de long sur $45 \mu$ de large, elle est cylindrique et un peu à gauche du plan sagittal. Elle atteint antérieurement à peu près la limite entre les deux derniers tiers de la longueur du corps. Chez les spécimens montés in toto, on voit à travers la paroi musculaire de la poche, un tissu glandulaire où serpente un tube plusieurs fois replié, qui est la vésicule séminale continuée par la pars prostatica. Celle-ci se termine par le canal éjaculateur court, qui s'ouvre dans le sinus génital à côté de la protubérance plus ou moins conique dite "Geschlechtszapfen ", qui est ici assez grosse.

Le sinus s'ouvre à l'extérieur par le pore génital, sur la face ventrale, un peu en avant de l'extrémité postérieure du corps. Au voisinage du pore génital il y a un amas de cellules glandulaires qui ont pour rôle de sécréter l'enveloppe du spermatophore. N'ayant eu à ma disposition que quelques individus immatures à utérus non développé, je ne peux rien dire des autres parties de l'appareil génital.

Chez nos échantillons, l'appareil excréteur n'est pas bien visible : tout au plus distingue-t-on, derrière la poche du cirre, un canal s'ouvrant à l'extrémitè postérieure du corps.

A ma connaissance, il a été décrit seulement cinq espèces de Prosorhynchus: P. squamatum Odhner, 1905 (= Gasterostoma armatum Olsson, 1868 et Levinsen, 1881) ; P. crucibulum (Rudolphi, 1819) ; P. aculeatum Odhner, 1905 (= Gasterostoma crucibulum van Beneden, $1870=$ Gasterostoma armatum Olsson, 1876) ; P. triglæ Nicoll, 1914 ; P. uniporus Ozaki, 1924.

Je résume comparativement leurs caractéristiques dans le tableau ci-joint (p. 558-559).

Mon Prosorhynchus de Siniperca ne peut être rapporté à aucune de ces cinq espèces, en raison de sa triple couronne d'épines, de l'extension du sac intestinal jusqu'à la concavité de l'arc des vitellogènes, de la forme des testicules, de la position de l'ovaire dorsalement au tube digestif, au niveau du milieu de la longueur de celui-ci.

Chez $P$. uniporus, seule espèce à vessie ne s'ouvrant pas directement à l'extérieur, mais dans le sinus génital, le pharynx est situé 
à l'union des deux derniers quarts de la longueur du corps comme chez $P$. aculeatum ; chez $P$. squamatum, $P$. crucibulum, $P$. triglæ (1) il est situé à peu près au milieu de la longueur du corps ; chez mon espèce il est dans la partie postérieure du tiers moyen.

Il est évident que, par suite du développement de l'utérus, les rapports des organes doivent être un peu différents, chez les individus complètement adultes, de ce qu'ils sont chez mes individus immatures ; cependant, j'estime que mon Prosorhynchus se sépare suffisamment des autres espèces du genre pour être regardé comme une espèce distincte. (2)

\section{BiBLIOGRAPHIE}

VAn Beneden. - Les poissons des côtes de Belgique, leurs parasites et leurs commensaux. Mém. Acad. roy. de Belg., XXXVIII, 1870, p. 1-100, pl. VI.

Dollfus (R.-Ph.). - Helmintha I (Trematoda et Acanthocephala). Faune des Colonies françaises, III, fasc. 2, 1929, p. 6-114, fig. 1-23.

Lühe (M.). - Trematoden. Süsswasserfauna Deutschlands, 1909.

Nicoll (W.). - On the entozoa of fishes from the Firth of Clyde. Parasitology, III, 1910, p. 322-359, pl. XXIX.

- Trematode parasites of fish from the English Channel. Journ, mar. biol. Ass. Plymouth, N. S., X, 1914, p. 466-505, fig. 1-6.

Onhner (T.). - Die Trematoden des arktischen Gebietes. Fauna arcticu, IV, 1905, p. 291-372, 4 fig., pl. II-IV.

Ozaki (Y.). - Some Gasterostomatous Trematodes of Japan. Jap. Journ. of Zool., II, 1920 , p. 35-60, fig. 1-27.

WARd (H. B.). - Trematoda in WARd and Whipple. Fresh-water Biology, 1918.

Ziegler (H. E.). - Bucepinalus und Gasterostomum. Zeitschr. f. wiss. Zool., XXXIX, 1883, p. 537-571, pl. XXXII-XXXIII.

(1) P. trigla Nicoll a êté décrit d'après des individus immatures.

(2) Par les soins de R.-Pli. Dollfus, le type de Prosorhynchus vaneyi mihi a été déposé au Museum National d'Histoire naturelle de Paris (Laboratoire de M. le Professeur Gh. Gravier) le 22 octobre 1930. No du registre d'entrées : 1930, n 11. 